\title{
Enriching the Interventional Vision of Cancer with Fluorescence and Optoacoustic Imaging
}

\author{
P. Beatriz Garcia-Allende, Jürgen Glatz, Maximilian Koch, and Vasilis Ntziachristos \\ Institute for Biological and Medical Imaging, Technische Universität München and Helmholtz Zentrum München, Munich, Germany
}

\begin{abstract}
Among several techniques considered for surgical and endoscopic imaging, novel optical methods are evolving as a promising approach for interventional guidance. Pilot clinical applications of fluorescence molecular imaging have demonstrated the benefits of using targeted fluorescent agents in cancer surgery. This premise can be extended broadly to interventional guidance through an increasing number of targeted agents and detection techniques. Beyond epi-illumination fluorescence imaging, optoacoustic (photoacoustic) methods are emerging to offer high-resolution crosssectional optical imaging through several millimeters to centimeters of depth. We present an overview of key recent developments in optical interventional imaging and outline the potential for a paradigm shift in surgical and endoscopic visualization.
\end{abstract}

Key Words: fluorescence molecular imaging; optoacoustic imaging; photoacoustic imaging; surgery; endoscopy; fluorescent agents

J Nucl Med 2013; 54:664-667

DOI: 10.2967/jnumed.111.099796

Many imaging methods have been clinically introduced over the past $40 \mathrm{y}$, including CT, MR imaging, ultrasound, SPECT, and PET. Despite the wide contribution of these methods to whole-body imaging, their translation to surgical or endoscopic guidance remains challenging ( 1 ) because of their cost, size, use of ionizing radiation, or the need to enclose the patient to provide a tomographic image $(2,3)$. Correspondingly, portable ultrasound endoscopic imaging (4) and intraoperative sentinel-node detection with $\gamma$-ray imaging have been explored (5).

Despite progress in medical imaging technology, clinical decision making in surgery and endoscopy still relies largely on the visual inspection or palpation of tissues. However, the human eye is not a particularly potent detector. It cannot visualize under the tissue surface, and its sensitivity is limited by the color contrast between healthy tissue and disease. These deficiencies of human vision limit the delineation of tumor margins during surgery or lead to high miss rates during white-light endoscopy, possibly compromising patient prognosis and efficient treatment $(6,7)$.

We briefly present here optical and optoacoustic methods emerging as alternatives to human vision for interventional imaging. Focus is given to technologies that we believe can potentially soon affect clinical outcomes, and key shortcomings that require attention are outlined.

Received Oct. 16, 2012; revision accepted Mar. 11, 2013.

For correspondence or reprints contact: Vasilis Ntziachristos, Institute for Biological and Medical Imaging, Technische Universität München and Helmholtz Zentrum München,

Trogerstrasse 9 D-81675, Munich, Germany.

E-mail: v.ntziachristos@tum.de

Published online Apr. 4, 2013.

COPYRIGHT @ 2013 by the Society of Nuclear Medicine and Molecular Imaging, Inc.

\section{IMAGING INTRINSIC CONTRAST}

Optical contrast in tissues has diverse origins. Typically, it arises from spatial variations of tissue constituents and tissue morphology, such as spatial changes in cellular density, the index of refraction, the biochemical composition of various tissues or vascularization, blood concentration, and oxygenation state (8). Humans can perceive this contrast only as superficial color differences and the corresponding patterns generated by color contrast. Accordingly, different optical imaging methods have been under consideration to better use optical contrast mechanisms and improve disease identification.

Narrow-band imaging, for example, operates on illuminating tissue in the blue $(415 \mathrm{~nm})$ and green $(540 \mathrm{~nm})$ spectrum of light and obtains difference images that highlight subsurface blood vessels (9). The principle of operation relies on the higher penetration of light in tissue at $540 \mathrm{~nm}$, which makes deeper mucosal and submucosal vessels visible, whereas capillaries in the superficial mucosal are emphasized by the 415 -nm light (9). However, lack of significant clinical gains has restricted the disseminated use of this technique.

Intrinsic fluorescence (autofluorescence) differences between normal, dysplastic, and malignant tissues have also been considered for malignancy detection (10). Changes in autofluorescence typically arise because of changes in naturally occurring fluorochromes in tissues, such as nicotinamide adenine dinucleotide phosphate oxidase. Regardless, autofluorescence imaging has not yet shown a compelling performance in clinical detection and is not widely used in surgical or endoscopic detection, although it might become a valuable tool as part of a multimodal imaging scheme (10).

Cross-sectional optical imaging methods have also been considered for intraoperative guidance. Optical coherence tomography (11) yields cross-sectional tissue images based on light-scattering variations in different tissue elements at high resolution $(<10 \mu \mathrm{m})$ through 1-2 mm of depth. Endoscopic microscopy has also been achieved using flexible, fiber-bundle-based confocal endoscopes (12). However, the small field of view achieved by optical coherence tomography or confocal imaging currently keeps these methods experimental rather than mainstream clinical tools.

\section{FROM NUCLEAR TO FLUORESCENT AGENTS}

To improve on the limitations of intrinsic contrast, optical methods have used extrinsically administered agents, in particular for surgical applications, as recently reviewed (13). Organic fluorescent dyes such as fluorescein and its derivatives or indocyanine green have been extensively used for human studies. Fluorescent dyes can reveal vascularization and physiologic parameters such as perfusion or vascular leakage or can track lymphatics at depths of up to $2-3 \mathrm{~cm}$, presenting an alternative to nuclear imaging methods. For this reason, several surgical procedures have considered the use of fluorescence guidance, such as to gain tissue characterization contrast in hepatobiliary and pancreatic surgery (14) and to detect lymph 
nodes $(15,16)$. However, fluorescent dyes have not been shown ideal for cancer detection, mostly because of a lack of specificity.

Surgical and endoscopic optical imaging can significantly benefit from experiences available to nuclear imaging approaches. Many fluorescent agents with specificity to cancer hallmarks have been developed $(17,18)$. These agents can reveal anatomic, biochemical, physiologic, and molecular features of disease; however, there is no clinical experience with virtually any of these agents. Similarly to nuclear imaging agents, the application of fluorescent agents to in vivo imaging requires careful tuning of the agent properties and probe targeting, activation, pharmacokinetics, and photophysics (19). From experiences gained from navigating the nuclear imaging regulatory process to lessons learned with tissue targets and the clinical performance of PET or SPECT probes, there is significant information available that can accelerate the clinical propagation of fluorescence molecular imaging (FMI).

\section{BENEFITS AND PITFALLS OF FLUORESCENCE IMAGING}

FMI, that is, imaging of fluorescent agents targeting specific tissue biomarkers, is an emerging modality with significant promise for enhancing endoscopic and surgical vision. Similarly to autofluorescence imaging or narrow-band imaging, FMI offers a large field of view that matches the surgeon's or endoscopist's view. Therefore, it is ideally suited for seamless integration with the current clinical standard while solving 3 of its paramount limitations. First, fluorescence imaging enables subsurface visualization. Fluorescence recorded from tissues always originates within tissue. By using agents in the near-infrared range, events at multiple millimeters of depth can be visualizeda marked change over human vision or white light endoscopy. Second, selection of agents with good clearance characteristics can enhance the contrast and, consequently, the detection sensitivity over intrinsic contrast imaging methods. Third, the use of "engineered contrast" afforded by targeted agents can offer specificity superior to that of imaging intrinsic contrast, such as the nonspecific tissue discolorations typically guiding today's surgical and endoscopic vision. Moreover, a foreseen synergy could combine nuclear imaging for intraoperative navigation and fluorescence imaging for local high-resolution inspection for margins and locoregional microfoci of disease.

However, FMI still attains 2 important limitations. First, most of today's cameras used for detection of fluorescence operate in simple photographic or video mode and do not accurately report on the true emission intensity and the underlying agent concentration. This is because the signals captured are the coupled effect of fluorescence emission intensity modulated (attenuated) by the tissue optical properties and the depth of the recorded activity, as showcased in Figure 1 (20). In contrast to high-energy photons that attain low sensitivity to tissue properties, visible or near-infrared photons are strongly scattered and absorbed by tissues. These effects reduce the image resolution of conventional epi-illumination fluorescence imaging (photography) and lead to nonquantitative and possibly inaccurate measurements that may yield false-positives and false-negatives (20). Emerging solutions for this challenge include the development of multispectral approaches that can correct for the effect of optical properties and reduce the artifacts seen on conventional epi-illumination fluorescence approaches (20).

A second limitation of FMI regards its 2-dimensional, surfaceweighted operation. Even if fluorescence signals can be collected from depths of at least a few millimeters, only 2-dimensional images can be formed, whereby deeper signals are more attenuated and appear mixed with more superficial signals. To address these limitations, a novel modality is rapidly emerging, termed multispectral optoacoustic tomography (21), which is discussed in a later section.

\section{CLINICAL APPLICATIONS OF TARGETED AGENTS}

Despite the challenges of the clinical translation of optical agents, the first human use of tumor-specific fluorescent agents for surgical visualization of ovarian cancer patients has been demonstrated (22). The clinical study used a real-time multispectral imaging system, approved for clinical use by the technical department of the University Medical Center Groningen. Folate conjugated to fluorescein isothiocyanate was intravenously administrated to ovarian cancer patients undergoing surgery for targeting the folate receptor- $\alpha$. Figure $2 \mathrm{~A} \mathrm{dem-}$ onstrates results from this study. Only a fraction of malignant lesions is visible on the color image. Conversely, fluorescent contrast, seen as pseudogreen color superimposed on the color image, detected up to 5-fold more lesions than did the surgeon's visual inspection (22).

Several other studies have confirmed FMI as a potent approach for interventional procedures. Bird-Lieberman et al. (23) investigated wheat germ agglutinin labeled with Alexa Fluor 680 (Invitrogen) as a candidate lectin to target cell-surface glycans altered in the progression of Barrett esophagus. The study imaged esophageal surgical specimens topically sprayed with the fluorescent lectin and demonstrated significant enhancement for the detection of dysplasia in Barrett esophagus, as shown in representative images in Figure 2B. The lectins used in this study are part of a regular diet and, depending on the particular type and concentration, are expected to have low toxicity.
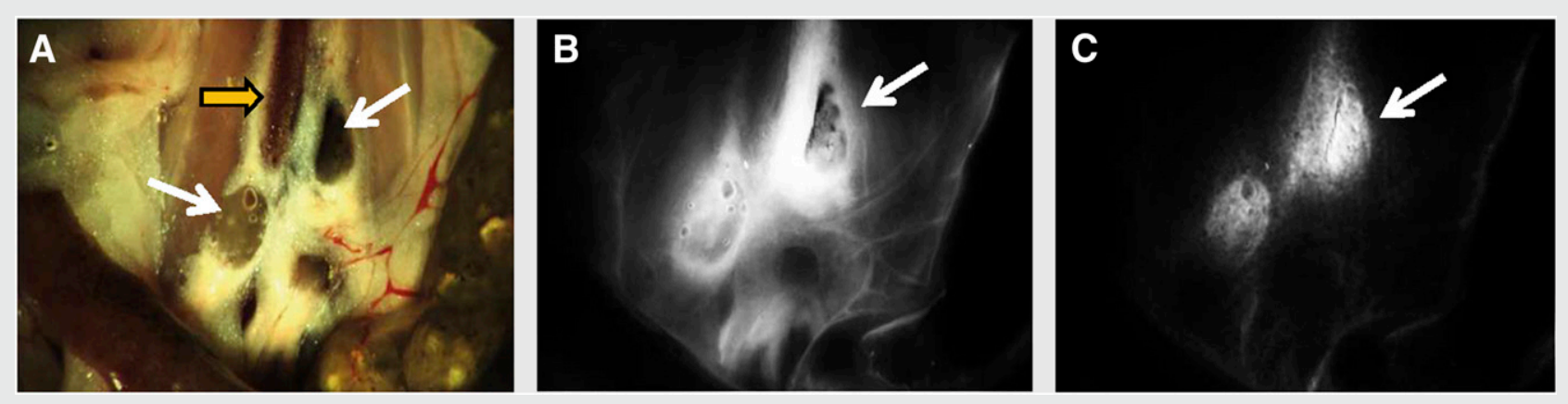

FIGURE 1. Effects of optical properties on fluorescence imaging demonstrated postmortem on surgically exposed mouse abdominal area. (A) Color fluorescence image showing lumbar lymph nodes (thin arrows) around inferior vena cava (thick arrow). Both lymph nodes were injected with identical mix of Indian ink and near-infrared fluorescent dye. (B) Conventional fluorescence image showing low signal intensity from lymph nodes, compared with bright background signals due to diffusion of fluorescence. (C) Fluorescence image corrected for optical properties showing markedly improved fluorescence quantification, correctly resolving underlying fluorescent activity in nodes. (Reprinted with permission from (20).) 
FIGURE 2. Representative images from van Dam et al. (22) (A) and Bird-Lieberman et al. (23) (B) that showcase enhanced cancer visualization in surgery and endoscopy through FMI. (A) From left to right: color and fluorescence imaging system placed above ovarian cancer patient prepared for surgery, color image acquired under white-light illumination, and color image with superimposed fluorescence (green). (B) From left to right: white-light image, fluorescence image before topical administration of fluorescent lectins, and fluorescence image after topical administration of fluorescent lectins, taken ex vivo with endoscope over segment of excised esophagus.

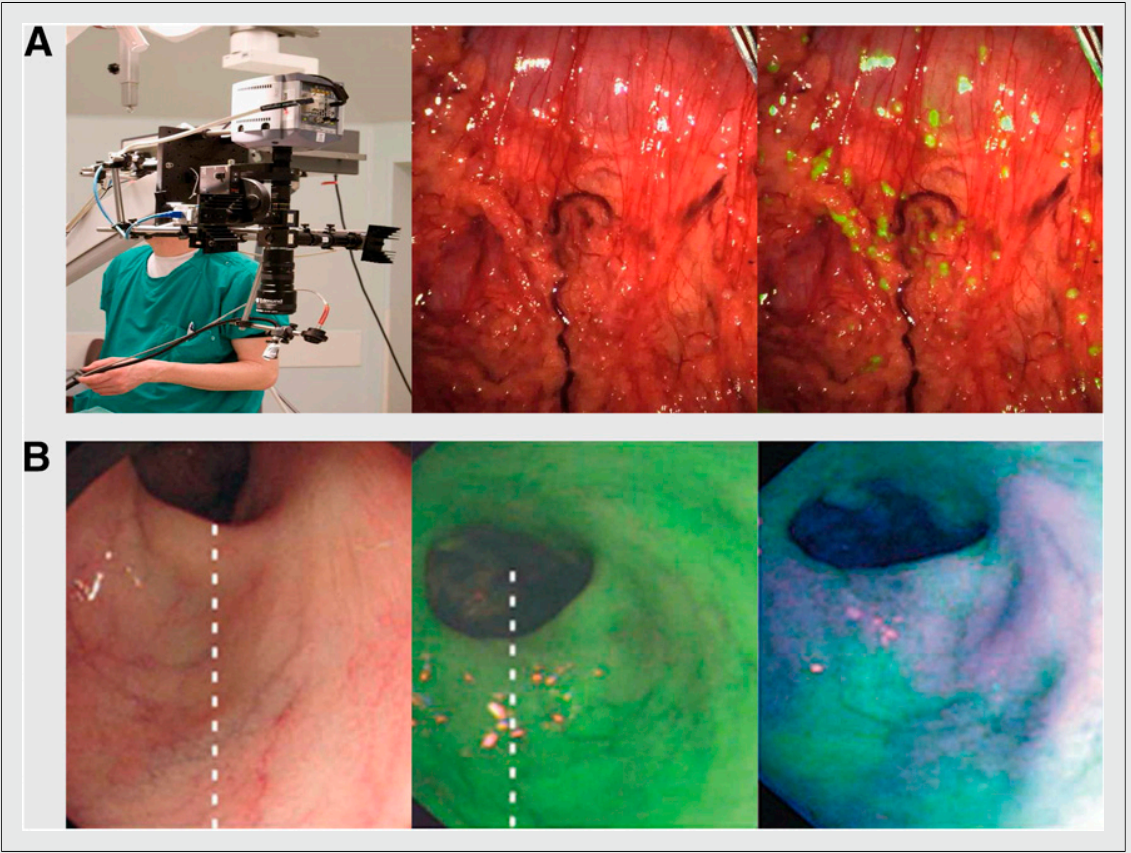

Topical spraying of fluorescent agents was alternatively considered by Urano et al. (24), who reported the use of a rapidly activatable fluorescent probe that is quickly cleaved by glutamyltranspeptidasespecific sequence, a cell-surface enzyme of glutathione metabolism that is highly expressed in various types of human cancer (24). The study demonstrated imaging of human peritoneal ovarian cancer in animal models, suggesting that the use of probes offering rapid activation of fluorescence can result in increased tumor-to-background ratios. Activatable probes may avoid the need for probe clearance, such as via tissue washing, which may be required when conventional fluorescent probes are topically applied, making the entire sprayed area fluorescent.

Topical application of fluorescent agents is considered an approach to reduce translational risk for new agents over the systemic administration of fluorescent agents. Conversely, systemic administration may offer better lesion targeting within the entire field of view, avoiding spraying inhomogeneity and better accessing more deeply seated lesions. Strategies to reduce the translational risk of systemically administered agents include the use of sensitive detectors to enable operation under microdosing and possibly the use of active

FIGURE 3. Optoacoustic vs. fluorescence imaging performed in vivo. (A) Photograph of mouse carrying subcutaneous tumor. (B) Cross-sectional multispectral optoacoustic tomography image along dotted line on $\mathrm{A}$. Gray-scale contrast shows high-resolution optical image at $750 \mathrm{~nm}$ through mouse abdomen. Superimposed in green pseudocolor is distribution of fluorescent dye indocyanine green through tumor and mouse abdomen. (C) Conventional epi-illumination fluorescence image shows typical performance of this type of imaging. (Adapted from (22).)

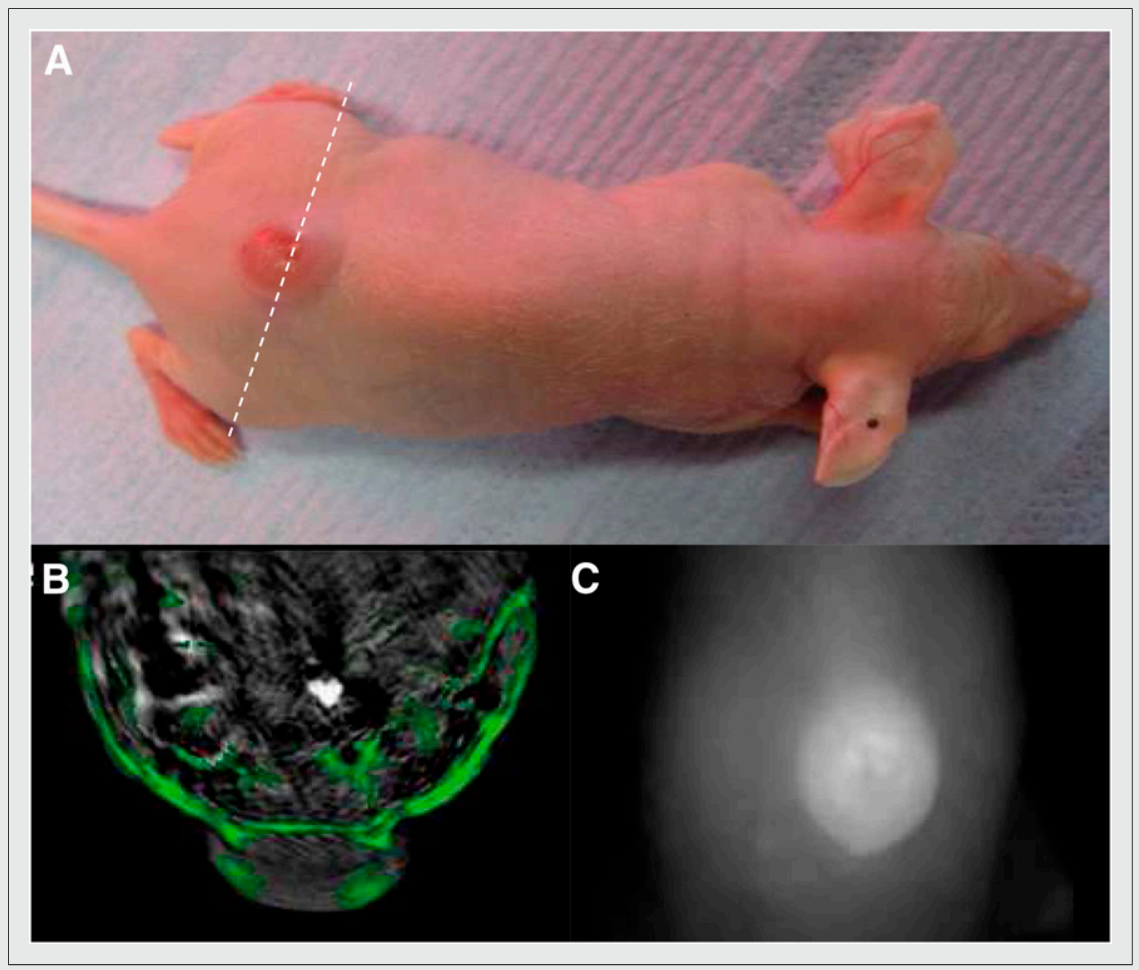


molecules with clinical experience, that is, drugs or nuclear imaging agents (17).

A notable optical imaging alternative with immediate clinical potential is Cerenkov luminescence imaging, CLI (25), which detects the Cerenkov emission of nuclear imaging tracers. Cerenkov radiation is the emission of a characteristic blue light that occurs when moving charged particles pass through a dielectric medium at speeds greater than the speed of light in that medium (25). Its use can enable optical imaging of clinically available nuclear imaging agents during surgery or endoscopy, a feature currently being evaluated in a clinical trial (ClinicalTrials. gov number, NCT01664936). Nevertheless, the low light intensity of the Cerenkov signal, combined with the short-lived radiotracers and the use of ionizing radiation, may limit routine clinical use.

These studies are far from an exhaustive list of the breadth of optical molecular imaging and are given as illustrations of recent progress toward clinical translation. Recent reviews give a more extended synopsis of preclinical studies using targeted optical contrast agents for cancer diagnostics (18).

\section{OPTOACOUSTIC IMAGING}

A powerful alternative emerging to address the FMI operational limitations is optoacoustic (photoacoustic) imaging, which allows cross-sectional optical imaging of tissues at resolutions ranging from a few tens to a few hundreds of microns (21). Optoacoustic imaging irradiates tissue with light of transient intensity (e.g., nanosecond photon pulses) and detects the ultrasonic waves generated within tissues in response to the absorption of the transient light energy and the corresponding minute temperature increase generated. When implemented as multispectral optoacoustic tomography, the technique sequentially illuminates tissue at multiple wavelengths and tomographically images intrinsic contrast (oxy- or deoxyhemoglobin, blood vessels) and exogenous photo-absorbing agents including fluorochromes and nanoparticles based on their distinct spectral signatures (21). Therefore, it offers a larger field of view, a higher penetration depth, and a wider ability to image intrinsic contrast and exogenous agents than does optical coherence tomography or confocal microscopy. Although the fluorochrome detection sensitivity of multispectral optoacoustic tomography is currently lower than that of FMI in detecting superficial fluorescence, the clinical application of optoacoustic imaging is expected to grow over the next few years to enable high-resolution cross-sectional imaging at depths ranging up to a few centimeters, as shown in Figure 3 (21).

\section{CONCLUSION}

For many years, FMI has been heralded as a potent tool for improving the endoscopic identification of cancer, reducing the number of incomplete surgeries by more sensitive identification of tumor margins, and enabling inspection of locoregional or lymph node metastasis. Recently, pilot clinical translation has confirmed the potential of FMI to enhance visual inspection, and advanced multispectral fluorescence and optoacoustic techniques are emerging for real-time guidance of interventional procedures. Similar to nuclear imaging, successful FMI translation would depend on the identification of potent biomarkers and targeting agents addressing unmet clinical needs, especially since promising results in animal studies do not necessarily imply clinical success. Challenges associated with regulatory processes in the clinical translation are also present, and FMI should follow the example of nuclear imaging and benefit from experiences built within.

\section{DISCLOSURE}

The costs of publication of this article were defrayed in part by the payment of page charges. Therefore, and solely to indicate this fact, this article is hereby marked "advertisement" in accordance with 18 USC section 1734. Financial support was received from the European Union within the 7th European Community Framework Program: ERC Advanced Investigational Award, DFG SFB824, and a Marie Curie Intra European Fellowship. No other potential conflict of interest relevant to this article was reported.

\section{ACKNOWLEDGMENT}

We are thankful to Karin M. Jentoft for useful discussions.

\section{REFERENCES}

1. Keereweer S, Kerrebijn JDF, van Driel PBAA, et al. Optical image-guided surgery: Where do we stand? Mol Imaging Biol. 2011;13:199-207.

2. Pickhardt PJ. Computed tomography colonography: emerging evidence to further support clinical effectiveness. Curr Opin Gastroenterol. 2013;29:55-59.

3. Liang D, Schulder M. The role of intraoperative magnetic resonance imaging in glioma surgery. Surg Neurol Int. 2012;3:S320-S327.

4. Tierney WM, Adler DG, Chand B, et al. Echoendoscopes. Gastrointest Endosc. 2007;66:435-442.

5. Vermeeren L, Meinhardt W, Bex A, et al. Paraaortic sentinel lymph nodes: toward optimal detection and intraoperative localization using SPECT/CT and intraoperative real-time imaging. J Nucl Med. 2010;51:376-382.

6. Fitzal F, Riedl O, Jakesz R. Recent developments in breast conserving surgery for breast cancer patients. Langenbecks Arch Surg. 2009;394:591-609.

7. Stoffel EM, Turgeon DK, Stockwell DH, et al. Missed adenomas during colonoscopic surveillance in individuals with Lynch syndrome (hereditary nonpolyposis colorectal cancer). Cancer Prev Res (Phila). 2008;1:470-475.

8. Kanick SC, van der Leest C, Djamin RS, et al. Characterization of mediastinal lymph node physiology in vivo by optical spectroscopy during endoscopic ultrasound-guided fine needle aspiration. J Thorac Oncol. 2010;5:981-987.

9. Song LMWK, Adler DG, Conway JD, et al. Narrowband imaging and multiband imaging. Gastrointest Endosc. 2008;67:581-589.

10. Song LMWK, Banerjee S, Desilets D, et al. Autofluorescence imaging. Gastrointest Endosc. 2011;73:647-650.

11. Robles FE, Wilson C, Grant G, et al. Molecular imaging true-colour spectroscopic optical coherence tomography. Nat Photonics. 2011;5:744-747.

12. Sonn GA, Jones SE, Tarin TV, et al. Optical biopsy of human bladder neoplasia with in vivo confocal laser endomicroscopy. J Urol. 2009;182:1299-1305.

13. Ntziachristos V, Soo JS, van Dam GM. Current concepts and future perspectives on optical imaging in cancer. J Biomed Opt. 2010;15:066024_1-066024_11.

14. Aoki T, Murakami M, Yasuda D, et al. Intraoperative fluorescent imaging using indocyanine green for liver mapping and cholangiography. J Hepatobiliary Pancreat Sci. 2010;17:590-594.

15. Troyan SL, Kianzad V, Gibbs-Strauss SL, et al. The Flare ${ }^{\mathrm{TM}}$ intraoperative nearinfrared fluorescence imaging system: a first-in-human clinical trial in breast cancer sentinel lymph node mapping. Ann Surg Oncol. 2009;16:2943-2952.

16. Crane LM, Themelis G, Arts HJ, et al. Intraoperative near-infrared fluorescence imaging for sentinel lymph node detection in vulvar cancer: first clinical results. Gynecol Oncol. 2011;120:291-295.

17. Scheuer W, van Dam GM, Dobosz M, et al. Drug-based optical agents: infiltrating clinics at lower risk. Sci Transl Med. 2012;4:134ps11.

18. Hellebust A, Richards-Kortum R. Advances in molecular imaging: targeted optical contrast agents for cancer diagnostics. Nanomedicine. 2012;7:429-445.

19. Hildebrand SA, Weissleder R. Near-infrared fluorescence: application to in vivo molecular imaging. Curr Opin Chem Biol. 2010;14:71-79.

20. Themelis G, Yoo JS, Soh KS, et al. Real-time intraoperative fluorescence imaging system using light absorption correction. J Biomed Opt. 2009;14:064012.

21. Herzog E, Taruttis A, Beziere N, et al. Optical imaging of cancer heterogeneity with multispectral optoacoustic tomography. Radiology. 2012;263:461-468.

22. van Dam GM, Themelis G, Crane LM, et al. Intraoperative tumor-specific fluorescent imaging in ovarian cancer by folate receptor- $\alpha$ targeting. Nat Med. 2011;17:1315-1319.

23. Bird-Lieberman EL, Neves AA, Lao-Sirieix P, et al. Molecular imaging using fluorescent lectins permits rapid endoscopic identification of dysplasia in Barrett's esophagus. Nat Med. 2012;18:315-321.

24. Urano Y, Sakabe M, Kosaka N, et al. Rapid cancer detection by topically spraying a $\gamma$-glutamyltranspeptidase-activated fluorescent probe. Sci Transl Med. 2011; 3:110ra119.

25. Holland JP, Normand G, Ruggiero A, et al. Intraoperative imaging of positron emission tomographic radiotracers using Cerenkov luminescence emissions. $\mathrm{Mol}$ Imaging. 2011;10:177-186. 